\title{
石墨烯狭缝受限孔道中水分子的分子动力学模拟
}

\author{
赵梦尧＼cjkstart杨雪平＼cjkstart杨晓宁* \\ (南京工业大学化学化工学院, 材料化学工程国家重点实验室, 南京 210009)
}

\begin{abstract}
摘要: 石墨烯是一种具有广泛应用前景的纳米材料, 特别是由石墨烯片层自组装形成的二维纳米通道能够应 用于物质的过滤分离. 本文采用分子动力学模拟方法研究了原态石墨烯/羟基改性石墨烯狭缝孔道中水分子的 微观行为, 模拟计算了水的界面结构性质和扩散动力学性质, 所研究的石墨烯孔宽为0.6-1.5 nm. 模拟结果表 明, 在石墨烯狭缝孔道中, 水分子受限结构呈现层状分布, 在超微石墨烯孔道(0.6-0.8 nm)中水分子可形成特 殊的环状有序结构, 石墨烯表面可诱导产生特殊的水分子界面取向. 在石墨烯孔道中, 水分子的扩散运动低于 主体相水分子的扩散运动, 差基化石墨烯孔道可以促使水分子的扩散能力降低. 对于改性石墨烯狭缝孔道, 由 于羟基的作用, 水分子可以自发渗入 $0.6 \mathrm{~nm}$ 的石墨烯孔道内. 模拟所得到的受限水分子的动力学性质与水分子 在石墨烯孔道内的有序结构有关. 本文研究结果将有助于分析理解水分子通过石墨烯纳米通道的渗透机理, 为 设计基于石墨烯的纳米膜提供理论指导.
\end{abstract}

关键词：分子模拟; 石墨烯; 水; 受限结构

中图分类号: 0641

\section{Molecular Dynamics Simulation of Water Molecules in Confined Slit Pores of Graphene}

\author{
ZHAO Meng-Yao YANG Xue-Ping YANG Xiao-Ning* \\ (State Key Laboratory of Materials-Oriented Chemical Engineering, College of Chemistry and Chemical Engineering, \\ Nanjing Tech University, Nanjing 210009, P. R. China)
}

\begin{abstract}
Graphene has potential applications in many fields. In particular, two-dimensional graphene nanochannels assembled from graphene sheets can be used for filtration and separation. In this work, molecular dynamics simulations were performed to investigate the microscopic structural and dynamical properties of water molecules confined in pristine and hydroxyl-modified graphene slit pores with widths of $0.6-1.5 \mathrm{~nm}$. The simulation results indicate that water molecules have layered structure distributions within the graphene nanoscale channels. The special ordered ring structure can be formed for water confined in the subnanometer pores $(0.6-0.8 \mathrm{~nm})$. Graphene surfaces are able to induce distinctive molecular interfacial orientations of water molecules. In the graphene slits, the diffusion of water molecules was slower than that in bulk water, and the hydroxyl-modified graphene pores could lead to more reduced water diffusion ability. For the hydroxyl-modified graphene pores, water molecules spontaneously permeated into the $0.6 \mathrm{~nm}$ slit pore. According to the simulation results, the dynamic behavior of confined water is associated with the ordered water structures confined within the graphene-based nanochannels. These simulation results will be helpful in understanding the penetration mechanism of water molecules through graphene nanochannels, and will provide a guide for designing graphene-based membrane structures.
\end{abstract}

\footnotetext{
Received: November 26, 2014; Revised: May 28, 2015; Published on Web: June 1, 2015.

"Corresponding author. Email: Yangxia@njut.edu.cn.

The project was supported by the National Natural Science Foundation of China (21376116).

国家自然科学基金(21376116)资助项目
} 
Key Words: Molecular simulation; Graphene; Water; Confined structure

\section{1 引言}

水在纳米尺寸环境(例如生物分子腔、沸石 膜、二氧化硅纳米孔 $)^{1-3}$ 的行为已引起广泛关注. 实 验和理论 ${ }^{4-11}$ 报道受限水具有不同于体态水的流动行 为和特殊结构. $\mathrm{X}$ 射线衍射实验 ${ }^{12}$ 发现水在温度为 148 到 $303 \mathrm{~K}$, 直径为 $1.13 \mathrm{~nm}$ 的碳纳米管下可以形成 像固体一样的结构. Koga等 ${ }^{13}$ 发现在压力 50-500 $\mathrm{MPa}$ 下, 水分子在直径为1.11-1.26 nm的椅型碳纳米 管中可以形成 $n$-多边形环状结构.

石墨烯具有大的比表面积、优越的电子性能 和机械性能等特点, 可用于超级电容器、光催化、 纳米过滤和盐水处理等. ${ }^{14-18}$ 石墨烯层状膜由单层石 墨烯堆砌而成, 具有纳米尺度的层间距, ${ }^{19,20}$ 这个特 殊的层间结构可以允许水分子通过，而截留其他分 子. $\mathrm{Han}^{\text {等 }}{ }^{21}$ 实验设计了带负电的超薄石墨烯纳米过 滤膜用于水的净化, 发现对有机染料具有高达 $99 \%$ 的截留率, 对于盐离子的截留率为 $20 \%-60 \%$, 同时 发现纯水渗透通过该石墨烯膜时具有很高的流率.

氧化石墨烯膜是石墨烯表面经过氧化的一种 特殊的石墨烯层状材料, 其主要制备方法有: 化学 氧化法和电化学氧化法. 氧化石墨烯表面存在多种 官能团, 如羟基、环氧基、羧基等, 而不同的氧化 制备工艺将会影响氧化石墨烯表面官能团的分布 结构及氧化程度. 由于氧化石墨烯制造工艺简单、 价格低廉, 且层间距具有可调节性等优点, 氧化石 墨烯引起广泛的关注. 在干燥条件下, 氧化石墨烯 膜层间距为 $\sim 0.3 \mathrm{~nm},{ }^{22}$ 在潮湿的空气或浸在水中时, 由于水化作用, 狭缝宽度可增加至 $1.0 \mathrm{~nm} .{ }^{23}$ Nair 等 ${ }^{24}$ 实验报道了水渗透通过氧化石墨烯膜未受阻碍, 而氦不能通过氧化石墨烯膜. Joshi等 ${ }^{23}$ 实验研究了 利用氧化石墨烯作为分离膜, 结果发现小离子可快 速渗透通过该膜. 近期研究 ${ }^{25-27}$ 表明, 使用不同官能 团对氧化石墨烯膜进行修饰，可以调节层间距，达 到分离废水中特定有机分子及离子的效果.

为了进一步开发上述石墨烯膜在实际过程中 的应用, 需更深入地了解水溶剂在原态石墨烯/亲水 性石墨烯层状狭缝纳米孔道中的相关性质. 由于现 有实验方法很难直接观测到如此小的受限空间内 分子行为, 分子模拟成为研究受限性质的有效方 法. ${ }^{28-31}$ 之前已有文献 ${ }^{4,21,23,24,32}$ 报道采用分子模拟方法 考察水分子通过石墨烯纳米通道时流动行为, 发现 水分子可以快速通过石墨烯孔道, 研究认为该现象 主要由于石墨烯表面原子与水分子非常弱的相互
作用能产生的, 但这些研究主要针对纳米尺度在大 于 $2.0 \mathrm{~nm}$ 的范围. 对于受限孔宽在0.6-1.5 nm的超微 孔中, 水分子结构和动力学行为将发生明显改变, ${ }^{33}$ 其 微观行为的研究仍然是一个前沿热点科学问题. Bocquet等 ${ }^{33}$ 发现当扶手椅式碳纳米管半径小于 0.5 $\mathrm{nm}$ 时, 管内水分子的结构性质发生改变可导致水分 子发生快速滑移现象, 这种小孔径石墨烯纳米孔道 非常适于水的渗透分离. 此外, 目前对两种不同亲 水特性的石墨烯孔道对受限水的作用缺乏深入的 研究, 因此, 考察水分子在0.6-1.5 nm的原态石墨烯/ 差基化石墨烯纳米通道内的结构特征与动力学性 质十分重要. 此外, 以前的模拟研究 28,3436 往往采用将 水分子直接嵌入孔道的周期性边界方法, 未考虑主 体相中水分子与孔内分子交互运动的影响, 从而可 能导致孔内水分子密度不是相应模拟体系下的自 然密度, 使模拟结果存在一定的差异.

本文采用分子动力学模拟的方法, 研究孔宽为 0.6-1.5 nm的原态石墨烯/亲水性石墨烯狭缝孔中, 水分子结构及动力学行为. 由于氧化石墨烯结构的 多样性和复杂性, 为了突出研究的关键问题, 在本 研究中, 通过直接的差基修饰来考察亲水性表面对 受限水分子的结构及动力学行为的影响. 在初始构 型中, 我们采用将石墨烯纳米狭缝孔道的两边与外 部主体水相(water reservoir)相连, 这样可以保持外 部水分子在纳米孔道内的水分子存在相互作用, 更 为接近真实体系下水分子穿越石墨烯狭缝孔道的 结构和动力学性质, 较为合理地反映实际膜孔道的 特征. 通过本文的研究, 可以进一步帮助认识受限 环境对水分子结构和运动的影响, 更为具体地了解 水分子在原态石墨烯/羟基化石墨烯狭缝孔道内的 结构性质及动力学行为的差异. 进而有助于对石墨 烯纳米通道的设计、改进, 为石墨烯膜在分离过程 中的应用提供理论指导.

\section{2 模拟方法}

\section{1 模拟模型}

模拟模型如图1所示, 从图中可以看出, 纳米通 道由两层构成, 每层是由3块石墨烯组成, 石墨烯之 间采用Bernal (ABA) 模型排布, ${ }^{37}$ 层与层之间的距离 为 $0.335 \mathrm{~nm} .{ }^{38,39}$ 石墨烯板 $x 、 y$ 方向的尺寸分别为 2.3332 和 $5.1048 \mathrm{~nm}$, 狭缝宽度 $H$ 分别为 $0.6 、 0.7$ 、 $0.8 、 0.9 、 1.0 、 1.5 \mathrm{~nm}$, 模拟盒子大小: $L_{x}=2.475$ $\mathrm{nm}, L_{y}=15.84 \mathrm{~nm}, L_{z}=6.912 \mathrm{~nm}$. 


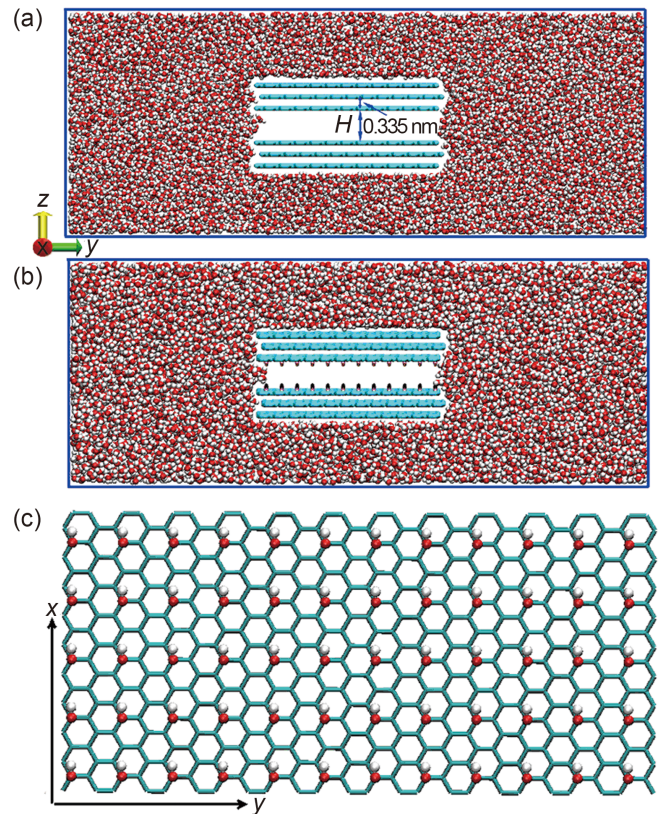

图1 模拟体系的构型

Fig.1 Configuration of the simulation system

(a) the pristine graphene slits; (b) the hydroxyl modified graphene slits; (c) the hydroxyl arrangement on the graphene surface; $H$ : slit pore width

本研究中水分子选取 $\mathrm{SPC} / \mathrm{E}^{40}$ 模型, 其中 $\mathrm{O}-\mathrm{H}$ 键的键长为 $0.1 \mathrm{~nm}, \mathrm{H}-\mathrm{O}-\mathrm{H}$ 的键角是 $109.47^{\circ}$, 模 拟过程中石墨烯的碳原子电荷采用中性处理, 其中 $\mathrm{C}-\mathrm{C}$ 的Lennard-Jones (LJ)相互作用是采用Cheng和 Steele ${ }^{41}$ 报道的参数. 羟基化石墨烯中的羟基直接连 接在石墨烯表面. $\mathrm{Xu}$ 等 ${ }^{42}$ 研究表明: 当羟基分布在石 墨烯平面时, 着基官能团呈随机分布状态与在表面 呈“晶格型”有序分布状态对计算所得的水分子润湿 角变化影响较小, 而在较为复杂的排布情况下, 如: 当羟基在石墨烯表面呈局部团簇排布时, 水分子的 润湿角可发生一定的变化, 该润湿行为的变化可能 影响水分子动力学行为. 为了减少复杂因素和突出 表面亲水特征, 我们采用文献 ${ }^{43}$ 中的处理方法来构 建一种亲水石墨烯模型, 即修饰的羟基官能团以矩 形 $(0.491 \mathrm{~nm} \times 0.425 \mathrm{~nm})$ 点阵结构在板面有序排列, 表面覆盖率近似为 $13 \%$, 羟基中的氧原子和氢原子 之间的距离 $\gamma_{\mathrm{OH}}=0.0945 \mathrm{~nm}$, 氧原子与被连接的碳原 子的距离 $\gamma_{\mathrm{CO}}=0.143 \mathrm{~nm}, \mathrm{OH}$ 键与石墨烯表面法向量 成 $71.5^{\circ}$ 夹角, 该 $\mathrm{OH}$ 取向角对应着普遍化OPLS力场 中的平衡位置的烷基醇 $\mathrm{C}-\mathrm{O}-\mathrm{H}$ 间的平衡夹角. ${ }^{44}$ 在模拟过程中, 采取将修饰官能团整体保持固定不 动的简化模型, 并以此考察亲水性表面对受限水分 子动力学性质的影响. 尽管这种亲水性石墨烯不能
表1 模拟使用的力场参数

Table 1 Force field parameters used in the simulation

\begin{tabular}{cccc}
\hline Pair interaction & $\varepsilon_{i i} /\left(\mathrm{J} \cdot \mathrm{mol}^{-1}\right)$ & $\sigma_{i /} / \mathrm{nm}$ & $q / e$ \\
\hline $\mathrm{C}_{\mathrm{g}}-\mathrm{C}_{\mathrm{g}}$ & 233.2 & 0.34 & 0.00 \\
$\mathrm{C}_{\mathrm{h}}-\mathrm{C}_{\mathrm{h}}$ & 233.2 & 0.34 & +0.265 \\
$\mathrm{O}_{\mathrm{h}}-\mathrm{O}_{\mathrm{h}}$ & 711.8 & 0.307 & -0.700 \\
$\mathrm{H}_{\mathrm{h}}-\mathrm{H}_{\mathrm{h}}$ & 0.000 & 0.00 & +0.435 \\
$\mathrm{O}_{\mathrm{w}}-\mathrm{O}_{\mathrm{w}}$ & 650.2 & 0.3166 & -0.8476 \\
$\mathrm{H}_{\mathrm{w}}-\mathrm{H}_{\mathrm{w}}$ & 0.00 & 0.00 & +0.4238 \\
\hline
\end{tabular}

$\mathrm{C}_{\mathrm{g}}$ : carbon atom in the pristine graphene, $\mathrm{C}_{\mathrm{h}}$ : carbon atom connected with hydroxyl group in modified graphene, $\mathrm{O}_{\mathrm{h}}$ : oxygen atom in hydroxyl group, $\mathrm{H}_{\mathrm{h}}$ : hydrogen atom in hydroxyl group, $\mathrm{O}_{\mathrm{w}}$ : oxygen atom in water, $\mathrm{H}_{\mathrm{w}}$ : hydrogen atom in water

完全代表氧化石墨烯, 但是其结果可以用来比较两 种不同石墨烯表面对受限水的作用, 类似的表面官 能团处理方式在以前文献 ${ }^{45,46}$ 中也有报道. 不同原子 之间的LJ相互作用参数采用的是Lorentz-Berthelot 混合规则, 具体模型参数如表1所示.

\section{2 模拟细节}

本文模拟使用LAMMPS软件, ${ }^{47}$ 选取NVT系综, 在温度为 298 K下考察原态石墨烯/羟基化石墨烯孔 道对水分子动力学性质的影响. 整个模拟过程中, 原态石墨烯/羟基化石墨烯浸在水主体相中, 并保持 整体结构固定. 运动方程采用velocity-Verlet算法求 解, 库仑相互作用采用particle-particle-particle-mesh (PPPM) 方法计算, 精确度为 $10^{-4}$, 非键范德华力采用 12-6 L-J势函数计算, 其L-J相互作用势能和静电相 互作用的截断半径都为 $1.2 \mathrm{~nm}$. 主体相中水分子个 数为 8204 , 密度控制为 $1.0 \mathrm{~g} \cdot \mathrm{cm}^{-3}$, 原态石墨烯/羟基 化石墨烯模拟体系中总原子个数分别为 27492 、 27732. 模拟采用三维周期性边界条件, 时间步长采 用 $1 \mathrm{fs}$, 总的模拟时间长达 $3 \mathrm{~ns}$.

\section{3 结果与讨论}

\section{1 受限水的结构性质}

\section{1 .1 密度分布}

首先我们考察了水分子渗入原态石墨烯/羟基 化石墨烯狭缝孔道中动力学行为. 图2所示为受限 孔道水分子数目随模拟时间的变化, 在原态石墨烯 狭缝孔道中, 当模拟时间达到 $100 \mathrm{ps}$ 时, 孔道内水分 子数目达到平衡, 当纳米孔宽为 $0.6 \mathrm{~nm}$ 时, 水分子不 能自发地进入石墨烯狭缝孔中, 这与文献 ${ }^{47}$ 报道相 一致, 这是由于憎水性微孔毛细管压力阻碍了水的 进入. 这也体现了水分子进入原态石墨烯狭缝孔道 

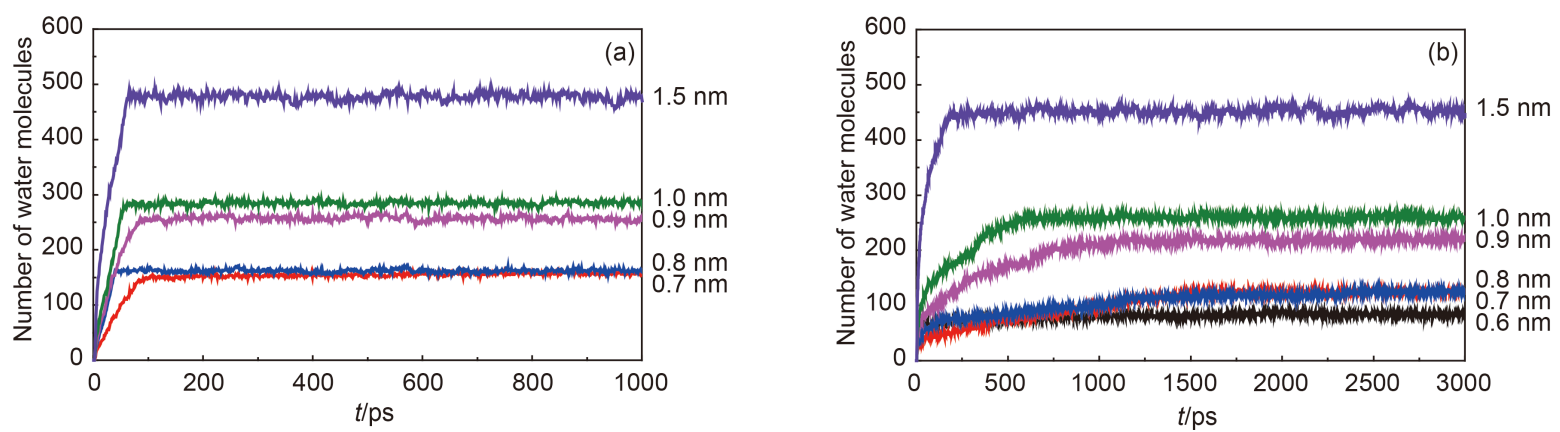

图2 狭缝孔中水分子数目随时间的变化

Fig.2 Changes of number of water molecules over time inside slit pores

(a) the pristine graphene slit pores; (b) the hydroxyl modified graphene slit pores

内获得的范德华能量不足以补偿其进入孔道内氢 键变化而损耗的能量. ${ }^{48,49}$ 随着孔宽的升高, 狭缝内 水分子数目增加; 对于羟基化石墨烯狭缝孔道, 在 着基的阻碍作用下, 水分子数目需更长的时间才能 达到平衡，并且由于差基的空间效应，孔道内水分 子的数目相比于同样原态石墨烯狭缝孔道要小. 不 过, 对于羟基化的狭缝, 在 $0.6 \mathrm{~nm}$ 的孔道内仍有水分 子自发渗入. 这是由该亲水性孔道毛细管推动力所 导致的，我们的结果与Boukhvalov等 ${ }^{50}$ 的报道相一 致.

水分子在原态石墨烯和羟基修饰石墨烯狭缝 孔中的密度分布见图3. 对于原态石墨烯狭缝孔道, 如图3(a)所示, 水的密度分布关于狭缝中心对称并 呈现出明显的层状结构, 在靠近碳板界面处存在明 显的流体减少, 同时在距离石墨烯表面 $0.32 \mathrm{~nm}$ 处, 呈现水密度最大峰. ${ }^{8.38}$ 并且由图可知, 在 $0.7 \mathrm{~nm}$ 孔中 形成单层的结构, $0.8 \mathrm{~nm}$ 中是介于单层与双层之间 的过渡层, 0.9 和 $1.0 \mathrm{~nm}$ 都是出现双层结构, $1.5 \mathrm{~nm}$ 出 现了四层结构. 出现不同分层结构不仅与狭缝宽度 有关同时还与 $\sigma_{\mathrm{CO}}$ 有关, 通过我们研究发现层状结构 与狭缝宽度存在如下近似关系:

$$
H=2 \sigma_{\mathrm{CO}}+(N-1) \sigma_{\mathrm{OO}}
$$

式中 $\sigma_{\mathrm{CO}}=0.3283 \mathrm{~nm}, \sigma_{\mathrm{OO}}=0.3166 \mathrm{~nm}$. 其中 $H$ 是狭缝 宽度, $N$ 是在狭缝中水形成的层数. 由此近似方程可 估算形成单层和双层狭缝宽度分别是 0.66 和 0.97 $\mathrm{nm}$, 这与模拟的结果相一致, 同时可推测狭缝宽度 为 $1.5 \mathrm{~nm}$ 时能够形成四层结构, 这与 $1.5 \mathrm{~nm}$ 时的密 度分布图呈现四层相吻合. 对于 $0.7 \mathrm{~nm}$ 原态石墨烯 狭缝孔道, Najafi等 ${ }^{34}$ 与Hirunsit和Balbuena ${ }^{36}$ 采用与 本文相同的力场参数, 而水分子采取直接嵌入的周 期边界孔模型方法时, 得出水分子的层状数目为 2 ,
而Deshmukh等 ${ }^{51}$ 采用与本文类似的由主体相自发进 入狭缝孔道的模型方法时, 水分子同样形成单层分 布状态. 因此, 石墨烯狭缝孔道的不同方式对水分 子的受限结构存在一定的影响, 这些不一致的结果 反映了不同孔道模型导致了受限孔中流体有效密 度差异, 进而影响受限结构.

对于羟基化石墨烯纳米孔道, 从图3(b)可看出 水的密度分布同样关于狭缝中心对称并呈现出明 显的层状结构. 进一步对比分析可知, 当羟基化石 墨烯狭缝宽度为 $0.8 、 1.0 、 1.5 \mathrm{~nm}$ 时, 形成的水层数 目与原态石墨烯狭缝有所不同, 这主要由于羟基的 亲水性及狭缝有效宽度协同作用的结果. 值得注意 的是, 在 $1.0 \mathrm{~nm}$ 的羟基化石墨烯狭缝孔道内, 水分子 呈现三层分布状态. 而在相同孔宽条件下, Xu等 ${ }^{52}$ 采 用直接嵌入的方式模拟得出水分子在羟基化石墨 烯孔道内的层状数目却为 2 . 该模拟结果的差异可 能同样由于孔道模拟不同所致.

从水分子的结构模拟构型图3(c-f)可看出: 0.7 $\mathrm{nm}$ 原态石墨烯狭缝孔中水分子呈现单层分布, 并形 成四元环结构. ${ }^{5,36}$ 与原态石墨烯孔道不同, 在图 $3(\mathrm{~d}$, e)中, 差基化狭缝孔道内水分子形成单层分布, 通过 构型图可以发现: 在 $0.6 \mathrm{~nm}$ 改性的石墨烯狭缝孔道 中大多数水分子可形成四元环结构, 而在 $0.7 \mathrm{~nm}$ 改 性孔道中形成了六元环结构. ${ }^{5}$ 对于 $0.8 \mathrm{~nm}$ 的羟基改 性石墨烯狭缝孔, 水分子形成双层结构, 在构型图 3(f)中展示了第一层水分子结构, 尽管部分第二层 水分子显露在第一层水分子的上方，但整体上，我 们可以清楚观察到水分子在狭缝孔道内呈线性分 布方式. 对于其他尺度狭缝孔道没有出现特殊的 结构.

\section{1 .2 径向分布函数}



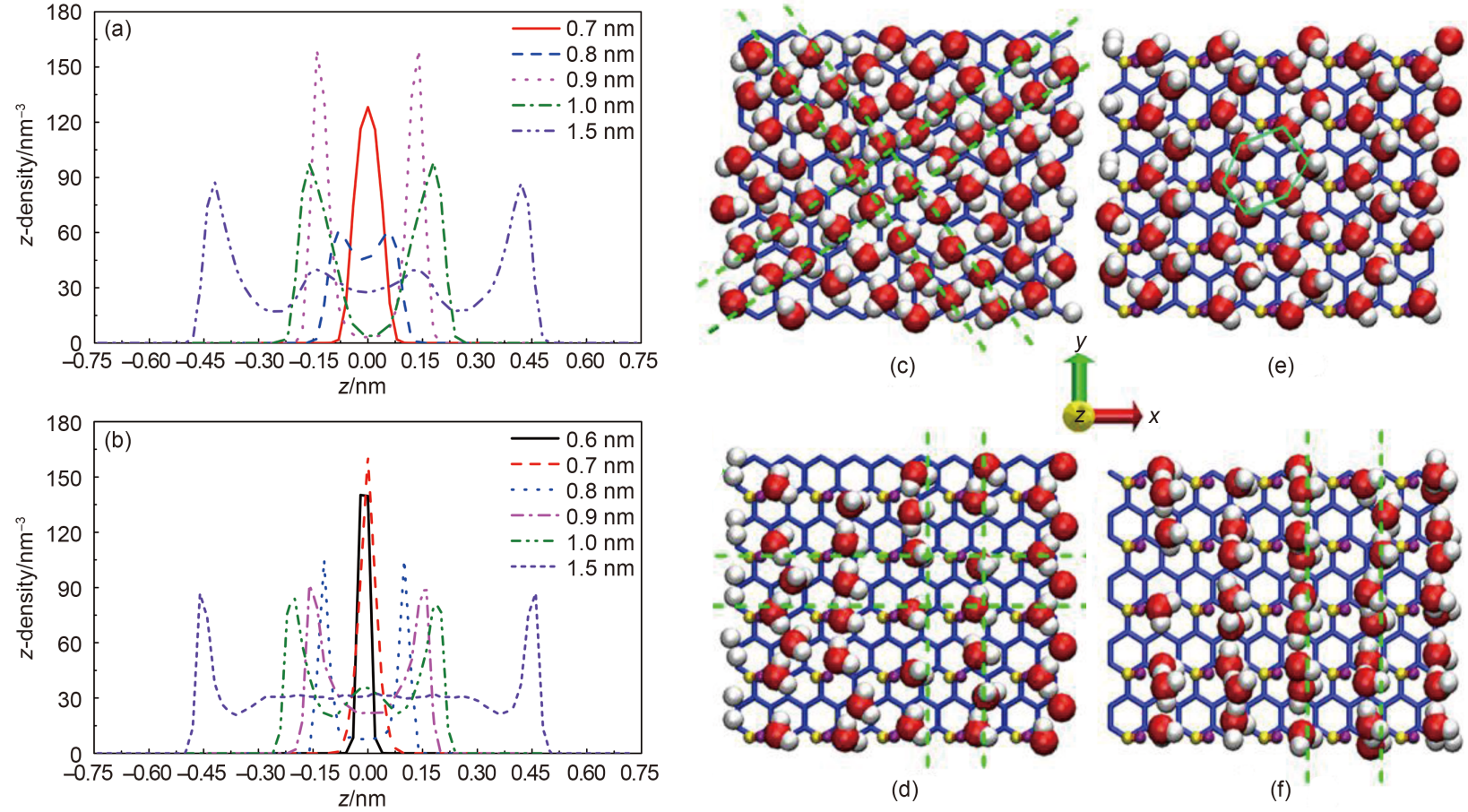

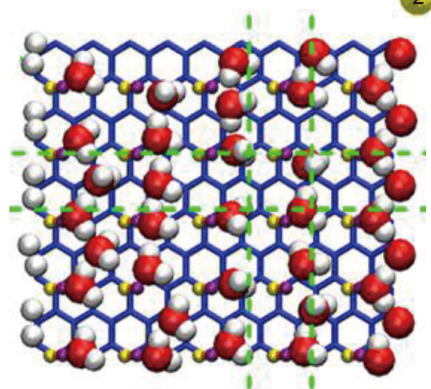

(d)

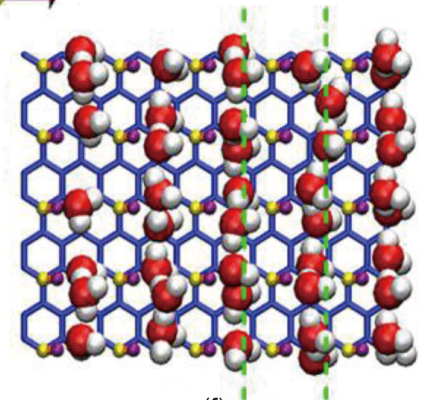

(f)

图3 受限水分子沿 $z$ 轴的密度分布图及在狭缝孔道内的构型图

Fig.3 Density profiles along the $z$-axis and the configurations inside the slit pores for confined water molecules

(a) the density profile for the pristine graphene slit pores; (b) the density profile for the hydroxyl modified graphene slit pores; (c) the configuration of confined water inside the pristine graphene slit pores with width of $0.7 \mathrm{~nm} ;(\mathrm{d}-\mathrm{f})$ the configurations of confined water inside the hydroxyl modified graphene slit pores with widths of $0.6,0.7,0.8 \mathrm{~nm}$, respectively.
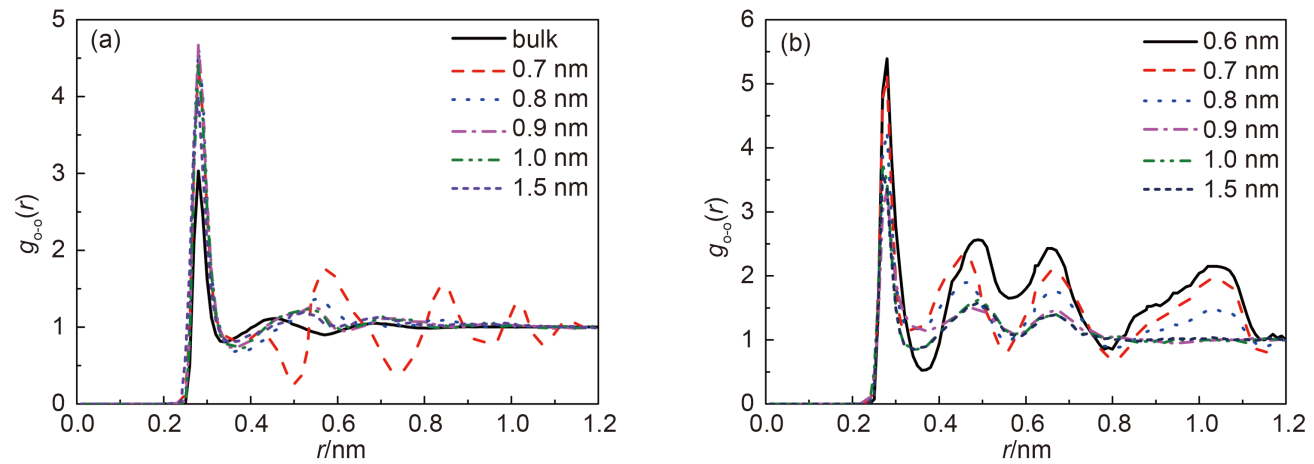

图4 不同狭缝孔道内靠近石墨烯板面水分子的径向分布函数 $\left(g_{0-0}(r)\right)$

Fig.4 Lateral radial distribution function $\left(g_{\text {o-o }}(r)\right)$ for the water molecules adjacent to the graphene wall in different slit pores

(a) the pristine graphene slit pores; (b) the hydroxyl modified graphene slit pores

径向分布函数 $(\mathrm{RDF})$ 是表征体系在原子水平上 结构性质的一种重要性质, 本文采用RDF分析固-液 界面层水的结构. 从图4(a)和4(b)中可以看出受限体 系和主体相中 $g_{\mathrm{o}-\mathrm{o}}(r)$ 的第一个峰大约在 $0.27 \mathrm{~nm}$ 处, 与文献 ${ }^{32,34}$ 报道的第一个峰位置相一致, 该峰的位置 对应于水分子之间第一配位圈距离, 此距离不受石 墨烯表面及其基团性质的影响. 根据图形可以看出 体态水的第二个比较宽的峰大约在 $0.45 \mathrm{~nm}$ 处, 其它
文献 ${ }^{40}$ 也得到了类似的结果, 而当 $H=0.8,0.9,1.0$, $1.5 \mathrm{~nm}$ 时第二个比较宽的峰大约在 $0.55 \mathrm{~nm}$ 处. 尤其 可以看出, 对于 $H=0.7 \mathrm{~nm}$, 发现接近界面水层的 $\mathrm{RDF}$ 表现为长程有序的特征, 这表明水在狭缝为 0.7 $\mathrm{nm}$ 的受限狭缝孔中形成一种类固态的结构. ${ }^{34}$ 从图 4(b)可以看出 $0.6 、 0.7 、 0.8 \mathrm{~nm}$ 道内, 水分子的 RDF也表现为长程有序的特征, 表明其在改性狭缝 孔中同样形成类似的固态结构, 这与图3(d-f)中所 
展现的构型图是一致的. 当羟基修饰的狭缝孔大于 $0.8 \mathrm{~nm}$ 时, RDF没有表现出长程有序的特征.

\subsection{3 取向分布}

为了进一步研究水分子在孔道内的分布状态, 我们分析水的 $\mathrm{OH}$ 键与表面法向量的夹角 $\varphi$, 以及偶 极方向与表面法向量的夹角 $\psi$ 来判定水分子在孔道 内的取向特征, 结果如图5所示. 取向分布显示, 在 原态狭缝孔道中, 水的 $\mathrm{OH}$ 键及水分子的偶极与 $z$ 轴 的夹角在约 $90^{\circ}$ 处达到最大值, 说明水分子以平行于 该石墨烯板的形式存在, 这将有利于受限孔中氢键 的形成, 这与之前 ${ }^{36,53}$ 研究结论相符. 从图中还可以 看出, 当石墨烯狭缝宽度大于 $0.9 \mathrm{~nm}$ 时, 由于水分子 的运动加剧, 取向分布的范围更广. 在羟基改性的 狭缝孔道中, 当孔宽为 $0.6 、 0.7 \mathrm{~nm}$ 时, 与原态石墨 烯狭缝孔道内水分子的羟基取向分布对比可以发 现, 水分子的 $\mathrm{OH}$ 键与 $z$ 轴的夹角在 $35^{\circ} 、 145^{\circ}$ 角度处 出现明显地凸起, 同时, 在对应的羟基化石墨烯狭 缝孔道中, 水分子的偶极与 $z$ 轴的夹角在 $90^{\circ}$ 处出现 最大值, 表明水分子的偶极方向平行于板面, 两种 取向结合可以说明: 当狭缝孔宽为 $0.6-0.7 \mathrm{~nm}$ 时, 相 比于原态石墨烯狭缝孔道, 在羟基功能化的石墨烯 狭缝孔道中, 更多水分子的 $-\mathrm{OH}$ 键倾向于指向改性
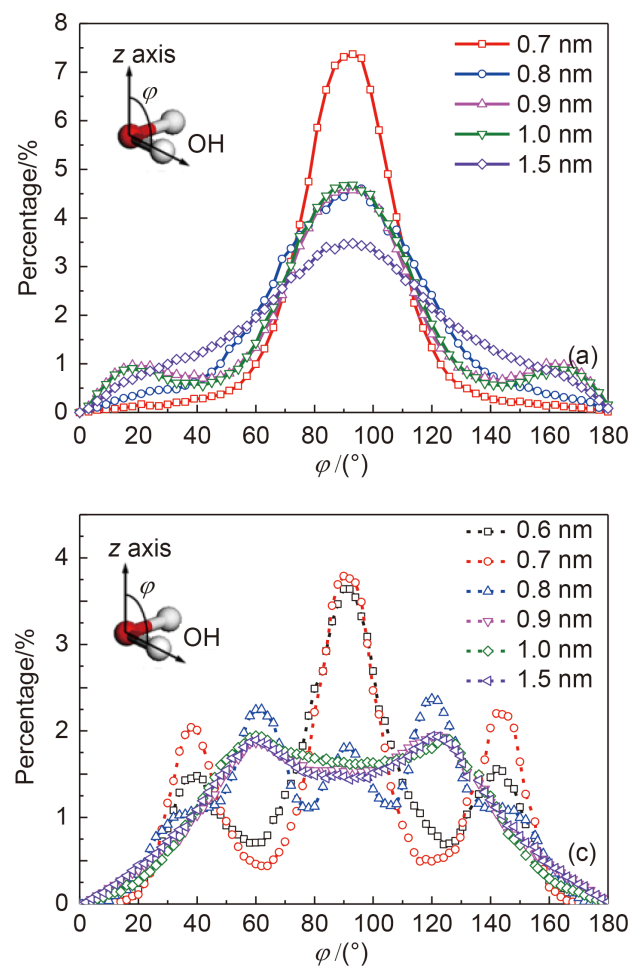

石墨烯表面, 主要由于部分水分子与羟基化石墨烯 表面的羟基可能形成氢键作用. 由此可知羟基的存 在影响水分子在狭缝孔道内的取向分布. 当狭缝宽 度超过 $0.7 \mathrm{~nm}$ 时, $\varphi$ 和 $\psi$ 都主要集中在 $60^{\circ}-120^{\circ}$ 之间, 取向角度分布变得相对无序.

\section{2 受限水的动力学性质}

\subsection{1 均方位移}

受限体系中水分子的动力学性质与体态水不 同. 因此, 我们进一步计算了平行于纳米通道方向 水的均方位移 $\left(\mathrm{MSD}_{x y}\right)$, 如图 6(a,b) 所示. 水的 $\mathrm{MSD}_{x y}$ 要比主体相要小, 表明微孔对其扩散施加一 定的限定作用. 基于 $\mathrm{MSD}_{x y}$ 的斜率, 可计算出主体相 的扩散系数是 $2.63 \times 10^{-9} \mathrm{~m}^{2} \cdot \mathrm{s}^{-1}$, 这与文献 ${ }^{10}$ 报道的 $\mathrm{SPC} / \mathrm{E}$ 水模型扩散系数 $\left(2.67 \times 10^{-9} \mathrm{~m}^{2} \cdot \mathrm{s}^{-1}\right)$ 有很好的 吻合. 水分子在原态石墨烯狭缝孔道中, 扩散系数 表现出非单调行为. 相比于其它狭缝孔道, 在 $H=0.8$ $\mathrm{nm}$ 的水分子呈现较大的扩散系数, 这可能由于该孔 道水分子受限密度较低所致. 在羟基化石墨烯狭缝 孔道中, 水分子的均方位移随着孔宽的增大呈上升 趋势. 由于羟基的亲水性及孔道内有效空间的变化, 水分子的扩散能力比相同宽度原态石墨烯狭缝孔 道低 $1-2$ 个数量级.
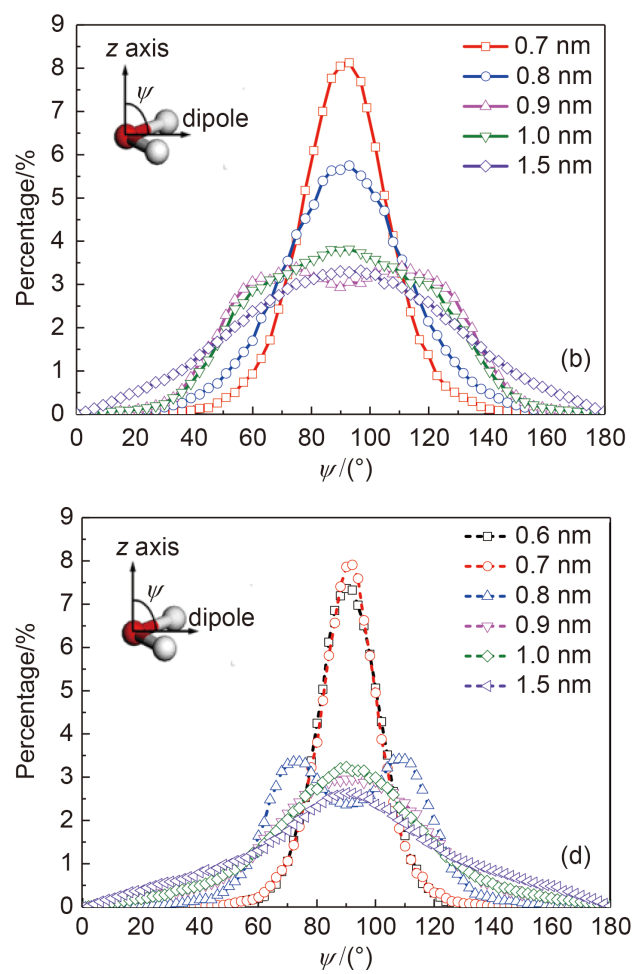

图5 受限水分子的取向角 $(\varphi, \psi)$ 分布

Fig.5 Orientation angle $(\varphi, \psi)$ distributions of confined water molecules

$(a, b)$ the pristine graphene slit pores; $(c, d)$ the hydroxyl modified graphene slit pores 

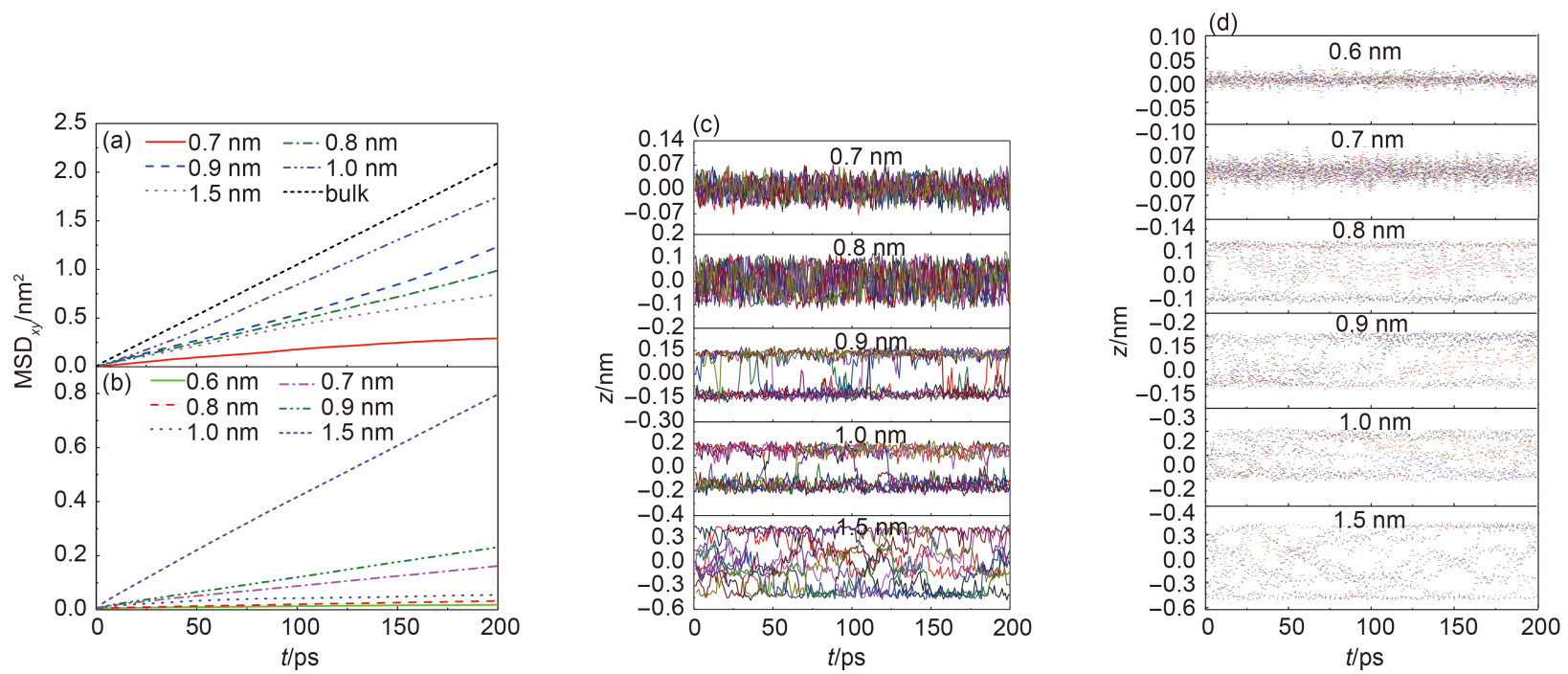

图6 (a,b)不同受限环境及主体相中多种类型水分子 $x y$ 平面的 $\mathrm{MSD}_{x y} ;(c, d)$ 受限水分子沿 $z$ 轴的运动轨迹

Fig.6 (a, b) Mean square displacement of $x y$ plane $\left(\right.$ MSD $\left._{x y}\right)$ for several typical water molecules in different confinement environments and bulk phase; $(c, d)$ trajectories of the confined water molecules along the $z$-axis

(a, c) the pristine graphene slit pores; (b, d) the hydroxyl modified graphene slit pores

对于受限水的运动性质, 本文进一步分析孔道 内水分子在 $z$ 方向的位置随时间的变化. 其图形 ${ }^{54,55}$ 主要根据输出的分子运动轨迹数据, 通过随机选取 受限孔道内一定水分子, 对其 $z$ 方向的位置随时间的 变化进行数据处理, 根据该图形(图6(c, d)), 我们可 以直接观察水分子沿垂直方向的运动行为随着孔 宽的变化. 从图形可知, 水在该方向的运动轨迹与 前面的密度分布与孔宽的变化行为一致, 表明其呈 现明显的层状结构, 并且随着狭缝孔宽的增大, 水 分子的运动变剧烈.

\section{2 .2 氢键}

分子在受限体系下的动力学性质不仅与液-固 之间的相互作用有关, 液-液之间的氢键 $(\mathrm{HB})$ 作用也 占主导地位, 图7 $(\mathrm{a}, \mathrm{b})$ 为水分子沿着表面法向 $(z$ 轴 )的氢键分布. 氢键的定义是依据几何标准 ${ }^{56}$ 提出的: $\mathrm{O} \cdots \mathrm{O}-\mathrm{H}$ 的夹角不超过 $30^{\circ}, \mathrm{O} \cdots \mathrm{O}$ 的距离不超过 $0.35 \mathrm{~nm}$. 从图 7 中可以看出氢键分布基本上关于狭 缝中心对称. 在原态体系中, 平均氢键数目介于 $3.13-$ 3.47 , 其中 $H=0.7 \mathrm{~nm}$ 形成的平均氢键数目最多, 而 $H=0.8 \mathrm{~nm}$ 形成的平均氢键数目最少. 对于 $H=0.7$ $\mathrm{nm}$, 从构型图可知形成四元环形结构从而容易形成 氢键, 而这种氢键网络结构不利于单个水分子在纳 米通道中热运动. ${ }^{34,57}$ 在 $0.8 \mathrm{~nm}$ 的纳米通道中水分子 间相对少的氢键数目对应其较大的扩散系数; 对于 羟基化石墨烯孔道, 在固-液界面层, 水分子之间形 成的氢键数目明显低于原态石墨烯孔道, 特别是在
小于 $0.9 \mathrm{~nm}$ 的羟基化石墨烯孔道. 这主要是由于表 面羟基与水分子更容易形成氢键，从而削弱了受限 水分子之间形成氢键的能力. 同时可以看出受限于 羟基修饰的石墨烯狭缝中水分子平均氢键数目明 显比主体相的平均氢键数目低, 随着狭缝宽度的增 大, 平均的氢键数目也相应增大.

\section{2 .3 氢键相关函数}

我们进一步分析了受限体系氢键稳定性的动 力学行为, 这里采用间歇性相关函数进行分析, 其 定义如下方程表示. ${ }^{58,59}$

$$
C_{\mathrm{HB}}(t)=\frac{\langle h(0) h(t)\rangle}{\langle h(0) h(0)\rangle}
$$

在式(2)中如果某两个水分子在 $t$ 时刻形成氢键 则 $h(t)=1$, 否则, $h(t)=0 . C_{\mathrm{HB}}(t)$ 能够反映氢键的结构 松弛动力学性质. 由图得知受限水的 $C_{\mathrm{HB}}(t)$ 衰减比主 体相水慢. 同时从图7(c)中发现狭缝宽度为 0.7 $\mathrm{nm}$ 时, $C_{\mathrm{HB}}(t)$ 衰减非常缓慢, 可认为主要是由于 0.7 $\mathrm{nm}$ 狭缝中水分子之间形成的四元环结构使得氢键 十分牢靠. 同样, 在羟基修饰石墨烯狭缝孔道中, 宽 度为 $0.6 、 0.7 、 0.8 \mathrm{~nm}$ 时, $C_{\mathrm{HB}}(t)$ 表现出类似的性质, 同时衰减更为缓慢. 总体看, 对于两种石墨烯表面 都表现为随着狭缝宽度的增大, 受限水的 $C_{\mathrm{HB}}(t)$ 衰减 变得越来越快, 这表明 $C_{\mathrm{HB}}(t)$ 的衰减很大程度上取决 于孔道宽度. $C_{\mathrm{HB}}(t)$ 衰减的越慢, 形成的氢键越稳定, 越不利于水分子在狭缝中的运动. 

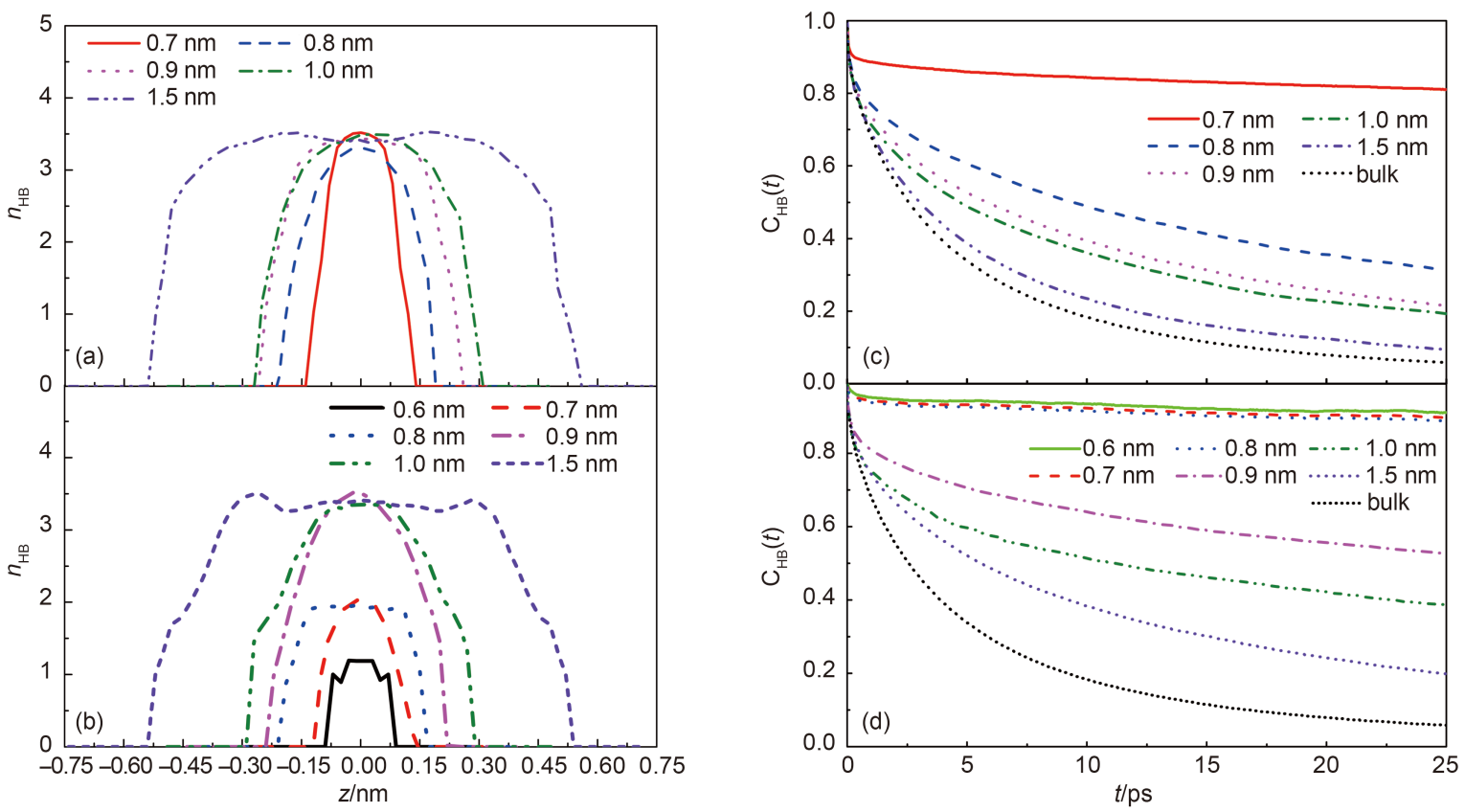

图7 (a, b) 狭缝孔道内水分子平均氢键数沿 $z$ 轴的分布; $(c, d)$ 受限水及体态水氢键的间歇性时间相关函数 $\left(C_{\mathrm{HB}}(t)\right)$

Fig.7 (a, b) Average number of hydrogen bond profiles along the $z$ axis for water inside slit pores; (c, d) the intermittent time correlation function $\left(C_{\mathrm{HB}}(t)\right)$ for the $\mathrm{H}_{2} \mathrm{O}-\mathrm{H}_{2} \mathrm{O}$ hydrogen bond numbers inside the nanochannnel as well as bulk water

$(\mathrm{a}, \mathrm{c})$ the pristine graphene slit pores; $(\mathrm{b}, \mathrm{d})$ the hydroxyl modified graphene slit pores

\section{4 结 论}

利用分子动力学模拟的方法, 研究了孔宽为 0.6-1.5 nm范围内, 原态石墨烯和羟基化石墨烯狭 缝孔道中水分子结构性质及动力学行为. 通过研究 发现水分子不能自发进入0.6 nm原态石墨烯狭缝孔 内, 而在同等宽度的羟基化狭缝中, 由于亲水性毛 细管推动力的作用, 水分子能够自发渗入. 通过密 度及取向分析, 狭缝孔内水分子形成明显的层状结 构, 并且在原态石墨烯狭缝孔道内水分子以平行于 碳板表面的形式存在，而在0.6-0.7 nm改性的石墨 烯狭缝孔道内, 部分水分子氢键指向碳板平面, 与 表面的羟基官能团形成氢键作用. 在超微 $(0.6-0.8$ $\mathrm{nm})$ 狭缝孔内, 水分子之间可以形成四元环、六元 环等类似固态一样的有序结构, 这种特殊构型促进 水分子之间形成氢键, 从而不利于水分子的扩散运 动. 其次, 在改性的石墨烯狭缝孔道内, 由于羟基的 亲水性及空间效应, 水分子的扩散能力比同等原态 石墨烯孔道要低1-2个数量级, 而在 $0.8 \mathrm{~nm}$ 原态石墨 烯狭缝孔道内, 由于水分子之间形成的平均氢键数 目最少, 水分子表现出相对较快的扩散能力. 相比 于主体相，在受限体系下，水分子之间形成的氢键 更稳定, 并且随着狭缝宽度的减小, 氢键的 $C_{\mathrm{HB}}(t)$ 衰 减越慢. 相比较, 羟基化石墨烯受限孔道中水分子
形成氢键结构稳定性更强. 综上所述, 石墨烯狭缝 孔道的尺寸效应及表面性质对受限水分子的结构 性质及动力学行为有着直接的影响.

\section{References}

(1) Pan, Y. S.; Birkedal, H.; Pattison, P.; Brown, D.; Chapuis, G. J. Phys. Chem. B 2004, 108 (20), 6458. doi: 10.1021/jp037219v

(2) Newsome, D. A.; Sholl, D. S. J. Phys. Chem. B 2005, 109 (15), 7239. doi: $10.1021 / \mathrm{jp} 044247 \mathrm{k}$

(3) Milischuk, A. A.; Ladanyi, B. M. J. Chem. Phys. 2011, 135 (17), 174709. doi: $10.1063 / 1.3657408$

(4) Qiao, Y.; Xu, X.; Li, H. Appl. Phys. Lett. 2013, 103 (23), 233106. doi: $10.1063 / 1.4839255$

(5) Han, S.; Choi, M. Y.; Kumar, P.; Stanley, H. E. Nat. Phys. 2010, 6 (9), 685. doi: $10.1038 /$ nphys 1708

(6) Du, F.; Qu, L. T.; Xia, Z. H.; Feng, L. F.; Dai, L. M. Langmuir 2011, 27 (13), 8437. doi: 10.1021/la200995r

(7) Strauss, I.; Chan, H.; Král, P. J. Am. Chem. Soc. 2014, 136 (4), 1170. doi: $10.1021 / \mathrm{ja} 4087962$

(8) Cicero, G.; Grossman, J. C.; Schwegler, E.; Gygi, F.; Galli, G. J. Am. Chem. Soc. 2008, 130 (6), 1871. doi: 10.1021/ja074418+

(9) Thomas, J. A.; McGaughey, A. J. H. Nano Lett. 2008, 8 (9), 2788. doi: $10.1021 / \mathrm{n} 18013617$

(10) Mashl, R. J.; Joseph, S.; Aluru, N. R.; Jakobsson, E. Nano Lett. 2003, 3 (5), 589. doi: 10.1021/n10340226

(11) Liu, Y. C.; Wang, Q.; Lü, L. H.; Zhang, L. Z. Acta Phys. -Chim. Sin. 2005, 21 (1), 63. [刘迎春, 王 琦, 吕玲红, 章连众. 物理化 
学学报, 2005, 21 (1), 63.] doi: 10.3866/ PKU.WHXB 20050113

(12) Iiyama, T.; Nishikawa, K.; Otowa, T.; Kaneko, K. J. Phys. Chem. 1995, 99 (25), 10075. doi: 10.1021/j100025a004

(13) Koga, K.; Gao, G. T.; Tanaka, H.; Zeng, X. C. Nature 2001, 412 (6849), 802. doi: 10.1038/35090532

(14) Stoller, M. D.; Park, S.; Zhu, Y. W.; An, J. H.; Ruoff, R. S. Nano Lett. 2008, 8 (10), 3498. doi: 10.1021/n1802558y

(15) Chandra, V.; Park, J.; Chun, Y.; Lee, J. W.; Hwang, I. C.; Kim, K. S. ACS Nano 2010, 4 (7), 3979. doi: 10.1021/nn1008897

(16) Zhang, H.; Lv, X. J.; Li, Y. M.; Wang, Y.; Li, J. H. ACS Nano 2010, 4 (1), 380. doi: 10.1021/nn901221k

(17) Cohen-Tanugi, D.; Grossman, J. C. Nano Lett. 2012, 12 (7), 3602. doi: 10.1021/nl3012853

(18) Hu, Y. J.; Jin, J.; Zhang, H.; Wu, P.; Cai, C. X. Acta Phys. -Chim. Sin. 2010, 26 (8), 2073. [胡耀娟, 金 娟, 张 卉, 吴 萍, 蔡称心. 物理化学学报, 2010, 26 (8), 2073.] doi: 10.3866/PKU. WHXB20100812

(19) Chen, H. Q.; Müeller, M. B.; Gilmore, K. J.; Wallace, G. G.; Li, D. Adv. Mater. 2008, 20 (18), 3557. doi: 10.1002/adma. 200800757

(20) Li, D.; Mueller, M. B.; Gilje, S.; Kaner, R. B.; Wallace, G. G. Nat. Nanotechnol. 2008, 3 (2), 101. doi: 10.1038/nnano. 2007.451

(21) Han, Y.; Xu, Z.; Gao, C. Adv. Funct. Mater. 2013, 23 (29), 3693. doi: 10.1002/adfm.v23.29

(22) Mi, B. X. Science 2014, 343 (6172), 740. doi: 10.1126/ science. 1250247

(23) Joshi, R. K.; Carbone, P.; Wang, F. C.; Kravets, V. G.; Su, Y.; Grigorieva, I. V.; Wu, H. A.; Geim, A. K.; Nair, R. R. Science 2014, 343 (6172), 752. doi: 10.1126/science.1245711

(24) Nair, R. R.; Wu, H. A.; Jayaram, P. N.; Grigorieva, I. V.; Geim, A. K. Science 2012, 335 (6067), 442. doi: 10.1126/ science. 1211694

(25) Sun, P. Z.; Zhu, M.; Wang, K. L.; Zhong, M. L.; Wei, J. Q.; Wu, D. H.; Xu, Z. P.; Zhu, H. W. ACS Nano 2013, 7 (1), 428. doi: 10.1021/nn304471w

(26) Sun, P. Z.; Zheng, F.; Zhu, M.; Song, Z. G.; Wang, K. L.; Zhong, M. L.; Wu, D. H.; Little, R. B.; Xu, Z. P.; Zhu, H. W. ACS Nano 2014, 8 (1), 850. doi: 10.1021/nn4055682

(27) Hu, M.; Mi, B. X. Environ. Sci. Technol. 2013, 47 (8), 3715. doi: $10.1021 / \mathrm{es} 400571 \mathrm{~g}$

(28) Xu, L.; Hu, Y. Z.; Ma, T. B.; Wang, H. Nanotechnology 2013, 24 (50), 505504. doi: 10.1088/0957-4484/24/50/505504

(29) Kolesnikov, A. I.; Zanotti, J. M.; Loong, C. K.; Thiyagarajan, P.; Moravsky, A. P.; Loutfy, R. O.; Burnham, C. J. Phys. Rev. Lett. 2004, 93 (3), 035503. doi: 10.1103/PhysRevLett. 93.035503

(30) Fernández-Serra, M. V.; Artacho, E. Phys. Rev. Lett. 2006, 96 (1), 016404. doi: 10.1103/PhysRevLett.96.016404

(31) Gao, W. X.; Wang, H. L.; Li, S. M. Acta Phys. -Chim. Sin. 2014, 30 (9), 1625. [高文秀, 王洪否, 李慎敏. 物理化学学报, 2014, 30 (9), 1625.] doi: 10.3866/PKU.WHXB201407031

(32) Xiong, W.; Liu, J. Z.; Ma, M.; Xu, Z. P.; Sheridan, J.; Zheng, Q.
S. Phys. Rev. E 2011, 84 (5), 056329. doi: 10.1103/ PhysRevE.84.056329

(33) Falk, K.; Sedlmeier, F.; Joly, L.; Netz, R. R.; Bocquet, L. Nano Lett. 2010, 10 (10), 4067. doi: 10.1021/n11021046

(34) Mosaddeghi, H.; Alavi, S.; Kowsari, M. H.; Najafi, B. J. Chem. Phys. 2012, 137 (18), 184703. doi: 10.1063/1.4763984

(35) Kumar, P.; Buldyrev, S. V.; Starr, F. W.; Giovambattista, N.; Stanley, H. E. Phys. Rev. E 2005, 72 (5), 051503. doi: 10.1103/ PhysRevE.72.051503

(36) Hirunsit, P.; Balbuena, P. B. J. Phys. Chem. C 2007, 111 (4), 1709. doi: $10.1021 / \mathrm{jp} 063718 \mathrm{v}$

(37) Warner, J. H.; Mukai, M.; Kirkland, A. I. ACS Nano 2012, 6 (6), 5680. doi: $10.1021 / \mathrm{nn} 3017926$

(38) Argyris, D.; Tummala, N. R.; Striolo, A.; Cole, D. R. J. Phys. Chem. C 2008, 112 (35), 13587. doi: 10.1021/jp803234a

(39) Liu, L.; Zhang, L.; Sun, Z. G.; Xi, G. Nanoscale 2012, 4 (20), 6279. doi: $10.1039 / \mathrm{c} 2 \mathrm{nr} 31847 \mathrm{~d}$

(40) Mark, P.; Nilsson, L. J. Phys. Chem. A 2001, 105 (43), 9954. doi: 10.1021/jp003020w

(41) Cheng, A.; Steele, W. A. J. Chem. Phys. 1990, 92 (6), 3858. doi: $10.1063 / 1.458562$

(42) Wei, N.; Lv, C. J.; Xu, Z. P. Langmuir 2014, 30 (12), 3572. doi 10.1021/1a500513x

(43) Janeček, J.; Netz, R. R. Langmuir 2007, 23 (16), 8417. doi: 10.1021/la700561q

(44) Cornell, W. D.; Cieplak, P.; Bayly, C. I.; Gould, I. R.; Merz, K. M.; Ferguson, D. M.; Spellmeyer, D. C.; Fox, T.; Caldwell, J. W.; Kollman, P. A. J. Am. Chem. Soc. 1995, 117 (19), 5179. doi: 10.1021/ja00124a002

(45) Gotzias, A.; Tylianakis, E.; Froudakis, G.; Steriotis, T. Microporous Mesoporous Mat. 2012, 154, 38. doi: 10.1016/ j.micromeso.2011.10.011

(46) Zhu, Y. D.; Guo, X. J.; Shao, Q.; Wei, M. J.; Wu, X. M.; Lu, L. H.; Lu, X. H. Fluid Phase Equilibr. 2010, 297 (2), 215. doi: 10.1016/j.fluid.2010.05.005

(47) Eun, C. S.; Berkowitz, M. L. J. Phys. Chem. B 2010, 114 (42), 13410. doi: $10.1021 /$ jp 1072654

(48) Lum, K.; Chandler, D.; Weeks, J. D. J. Phys. Chem. B 1999, 103 (22), 4570. doi: 10.1021/jp984327m

(49) Ren, X. P.; Zhou, B.; Wang, C. L. J. Chem. Phys. 2012, 137 (2), 024703. doi: $10.1063 / 1.4733719$

(50) Boukhvalov, D. W.; Katsnelson, M. I.; Son, Y. W. Nano Lett. 2013, 13 (8), 3930. doi: 10.1021/n14020292

(51) Deshmukh, S. A.; Kamath, G.; Baker, G. A.; Sumant, A. V.; Sankaranarayanan, S. K. R. S. Surf. Sci. 2013, 609, 129. doi: 10.1016/j.susc.2012.11.017

(52) Wei, N.; Peng, X. S.; Xu, Z. P. ACS Appl. Mater. Inter. 2014, 6 (8), 5877. doi: 10.1021/am500777b

(53) Pertsin, A.; Grunze, M. J. Phys. Chem. B 2004, 108 (4), 1357. doi: $10.1021 / \mathrm{jp} 0356968$

(54) Hub, J. S.; Winkler, F. K.; Merrick, M.; de Groot, B. L. D. J. Am. Chem. Soc. 2010, 132 (38), 13251. doi: 10.1021/ jp0356968 
(55) Zang, J.; Konduri, S.; Nair, S.; Sholl, D. S. ACS Nano 2009, 3 (6), 1548. doi: 10.1021/nn9001837

(56) Luzar, A.; Chandler, D. Nature 1996, 379 (6560), 55. doi: $10.1021 / j p 044247 \mathrm{k}$

(57) Striolo, A. Nano Lett. 2006, 6 (4), 633. doi: 10.1038/379055a0
(58) Martí, J.; Sala, J.; Guàrdia, E. J. Mol. Liq. 2010, 153 (1), 72. doi: 10.1016/j.molliq.2009.09.015

(59) Jorgensen, W. L.; Chandrasekhar, J.; Madura, J. D.; Impey, R. W.; Klein, M. L. J. Chem. Phys. 1983, 79 (2), 926. doi: $10.1063 / 1.445869$ 